Fabrication and current optical

performance of a large

diamond-machined ZnSe immersion grating

Y. Ikeda, N. Kobayashi, P. J. Kuzmenko, S. L. Little, C. Yasui, S. Kondo, H. Mito, K. Nakanishi, Y. Sarugaku

July 20, 2010

SPIE Modern Technologies in Space- and Ground-Based Telescopes and Instrumentation San Diego, CA, United States June 27, 2010 through July 2, 2010 
This document was prepared as an account of work sponsored by an agency of the United States government. Neither the United States government nor Lawrence Livermore National Security, LLC, nor any of their employees makes any warranty, expressed or implied, or assumes any legal liability or responsibility for the accuracy, completeness, or usefulness of any information, apparatus, product, or process disclosed, or represents that its use would not infringe privately owned rights. Reference herein to any specific commercial product, process, or service by trade name, trademark, manufacturer, or otherwise does not necessarily constitute or imply its endorsement, recommendation, or favoring by the United States government or Lawrence Livermore National Security, LLC. The views and opinions of authors expressed herein do not necessarily state or reflect those of the United States government or Lawrence Livermore National Security, LLC, and shall not be used for advertising or product endorsement purposes. 


\title{
Fabrication and current optical performance of a large diamond-machined ZnSe immersion grating
}

\author{
Yuji Ikeda ${ }^{a, e}$, Naoto Kobayashi ${ }^{b}$, Paul J. Kuzmenko ${ }^{c}$, Steve L. Little ${ }^{c}$, Chikako Yasui ${ }^{d}$, Sohei \\ Kondo $^{e}$, Hiroyuki Mito ${ }^{f}$, Kenshi Nakanishi ${ }^{e}$, and Yuki Sarugaku ${ }^{g}$ \\ ${ }^{a}$ Photocoding, 61-112 Iwakura-KitaIkeda-cho, Sakyo-ku, Kyoto 606-0004, Japan; \\ ${ }^{b}$ Institute of Astronomy, the University of Tokyo, 2-21-1 Osawa, Mitaka, Tokyo 181-0015, \\ Japan; \\ ${ }^{c}$ Lawrence Livermore National Laboratory, 7000 East Avenue, Livermore, CA 94550, USA; \\ ${ }^{d}$ National Astronomical Obsevatory of Japan, 2-21-1 Osawa, Mikata, Tokyo 181-8588, Japan; \\ ${ }^{e}$ Deprtment of Physics, Faculty of Science, Kyoto-Sangyo University, Motoyama, Kamigamo, \\ Kita-ku, Kyoto 603-8555, Japan;

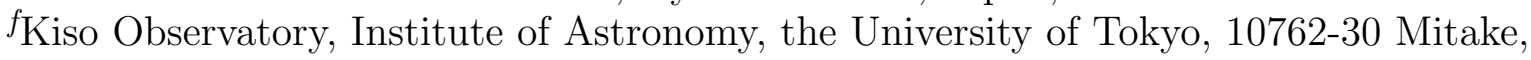 \\ Kiso-machi, Kiso-gun, Nagano 397-0101, Japan; \\ ${ }^{g}$ Japan Aerospace Exploration Agency, Institute of Space and Astronautical Science, 3-1-1 \\ Yoshinodai, Chuo-ku, Sagamihara, Kanagawa 252-5210, Japan;
}

\begin{abstract}
ZnSe immersion gratings provide the possibility of high resolution spectroscopy in the wide infrared wavelength region from the NIR (Near Infrared) to the MIR (Mid Infrared), because ZnSe has a high refractive index $(n \sim 2.45)$ and a low internal extinction in these wavelength regions. We are developing ZnSe immersion grating for a ground-based NIR high-resolution spectrograph and a space MIR high-resolution spectrograph. We already have produced fine grooves on the ZnSe flat substrate with a small pitch $(\sim 30 \mu \mathrm{m})$ using nano precision flycutting technique at the Lawrence Livermore National Laboratory, ${ }^{1}$ which satisfies our requirements even for the short NIR application. ${ }^{2}$

Our next step is to fabricate a large prism-shaped ZnSe immersion grating with this technology. The triangle prism has the entrance surface $50 \mathrm{~mm} \times 23 \mathrm{~mm}$ and the apex angle of $70 \mathrm{deg}$. Untile now, we tried three $\mathrm{R} \& \mathrm{D}$ cutting runs. We examined the optical performances of the immersion grating sample from the second cutting run, which showed the best performances. Although a lot of chipping are seen at the edge of the blaze by the microscopic observation, we found that the groove shape is quite good with the surface irregularity of $0.74 \lambda$ (pv) and the random pitch error of $5.2 \mathrm{~nm}(\mathrm{rms})$, which closely meet with our requirements. In the HeNe laser spectra taken under both grism and immersion configurations, strong ghosts were observed at the intermedium of the diffracted orders. These interorder ghosts may originate from the differences of the pitch and/or shape between odd and even grooves due to the cutting procedures. In addition, we also investigated a suitable reflectivecoating for the diffraction surface. As a result, we concluded that aluminum or cupper by suppering process is the best materials in the wavelength region of WINERED. Finally, we discuss the pssible improvement points and prospect for the next trial in this summer.
\end{abstract}

Keywords: Infrared, Spectroscopy, High resolution, Optical device, Immersion grating, nano technology

\section{INTRODUCTION}

Immersion grating is a powerful optical device for astronomical spectroscopy which is immersed into an optical material with the high refractive index of $n$. The maximum spectral resolution $\left(R_{\max }\right)$ of a spectrometer using

Further author information: (Send correspondence to Y.I.)

Y.I.: E-mail: ikeda@photocoding.com, Telephone: +81 757086120

N.K.: E-mail: naoto@ioa.s.u-tokyo.ac.jp, Telephone: +81 422345032 
the immersion grating is well known as,

$$
R_{\max }=\frac{\lambda}{\Delta \lambda}= \begin{cases}\frac{2 n \phi \tan \theta_{\mathrm{B}}}{D s} & \text { for the seeing limited condition, } \\ \frac{2 n \phi \tan \theta_{\mathrm{B}}}{\lambda} & \text { for the diffraction limited condition, }\end{cases}
$$

where $\phi$ is the collimated beam diameter which is deeply related to the overall instrumental volume, $\theta_{\mathrm{B}}$ is the blaze angle, $D$ is the telescope diameter, and $s$ is the silt width (e.g., see Yasui et al. 2006 ${ }^{3}$ ). From Eq.(1), immersion grating can provide $n$ times spectral resolution or can reduce the collimated beam, namely instrumental volume, into the size of $1 / n$, compared to classical reflective grating (see Figure.1). Thus, this grating received a lot of attention as a key device for high resolution spectrometer with $R \geq 70,000$ attached to the Extremely Large Telescope such as Thirty Meter Telescope ${ }^{4-6}$ and with $R \geq 30,000$ mounted on the spece telescope such as SPICA $^{7-10}$ because very large and heavy cryogenic instruments need to be built.

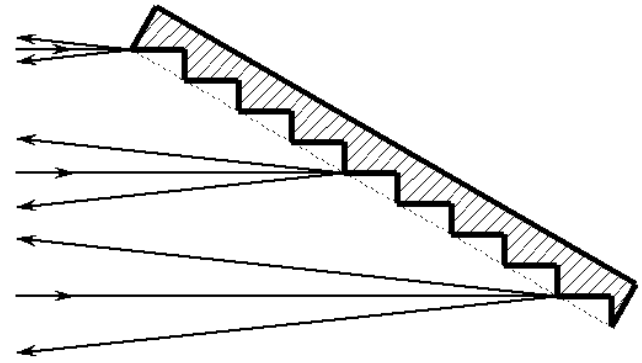

classical grating

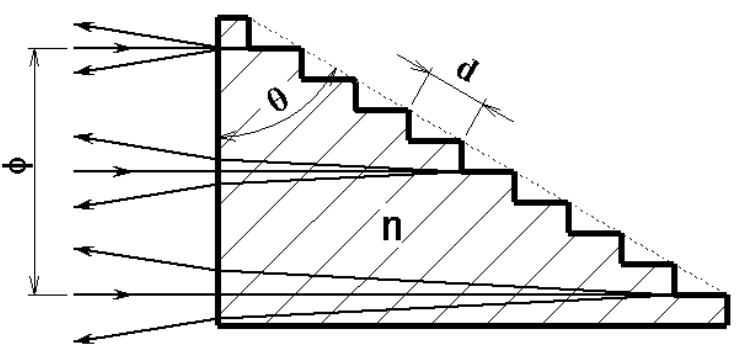

Immersion grating

Figure 1. A classical reflective grating and an immersion grating

We have been developing a high resolution spectrograph, WINERED,${ }^{11,12}$ which has the possibility of providing high resolution spectra with $R_{\max }=100,000$ at the short NIR wavelengths $(\lambda=0.9-1.35 \mu \mathrm{m} ; z$, $Y$, and $J$ bands) using a ZnSe or ZnS immersion grating. However, the immersion grating technology for the NIR wavelengths had not been established for a long time, except for silicon immersion grating fabricated by the photolithographic technique, ${ }^{13,14}$ because it was difficult to cut brittle materials, polycrystal CVD-ZnSe or CVD-ZnS *, without any chipping on the groove edge. Recently, we succeeded to produce fine grooves on the ZnSe substrate with a small pitch $(\sim 30 \mu \mathrm{m})$ by nano precision fly-cutting technique at the Lawrence Livermore National Laboratory, ${ }^{1}$ and we concluded that it achieves the good surface irregularity $(<\lambda / 8)$, the small surface roughness $(<5 \mathrm{~nm})$, and the small random pitch error $(<3 \mathrm{~nm})^{2}{ }^{2}$

Finally, we produced a large prism-shaped ZnSe immersion grating with an entrance aperture of $23 \mathrm{~mm} \times 55$ mm using this technology. In this paper, we report the optical performances of this prism grating and discuss the feasibility as an optical device for astronomical spectrometers. In section 2, we briefly describe the fabrication condition of the grating. In section 3, we report the results of the optical testings. In section 4, we discuss suitable reflective coatings on the diffraction surface of ZnSe for the short NIR region. And then, we summarize our results and describe the future prospects in the last section.

\section{MACHINING THE ZNSE PRISM GRATING}

We prepared a large triangle ZnSe prism with the entrance aperture of $23 \mathrm{~mm} \times 50 \mathrm{~mm}$ and the angles 90 , 70 , and $20 \mathrm{deg}$, supplied by II-VI Inc. The entrance face is slightly tilted to separate the front surface reflection from the diffraction beam (see Figure.2). The diffraction area is about $50 \mathrm{~mm} \times 58 \mathrm{~mm}$, on which the grooves with the pitch of $30 \mu \mathrm{m}$ and the blaze angle of $70 \mathrm{deg}$ were produced. For machining the grating, we used a nano precision fly-cutting technique with the Precision Engineering Research Lathe (PERL II) at Lawrence Livermore

\footnotetext{
*Only plycrystal CVD-ZnSe or CVD-ZnS is commercially available for large bulk with the thickness of $>50 \mathrm{~mm}$ at present.
} 

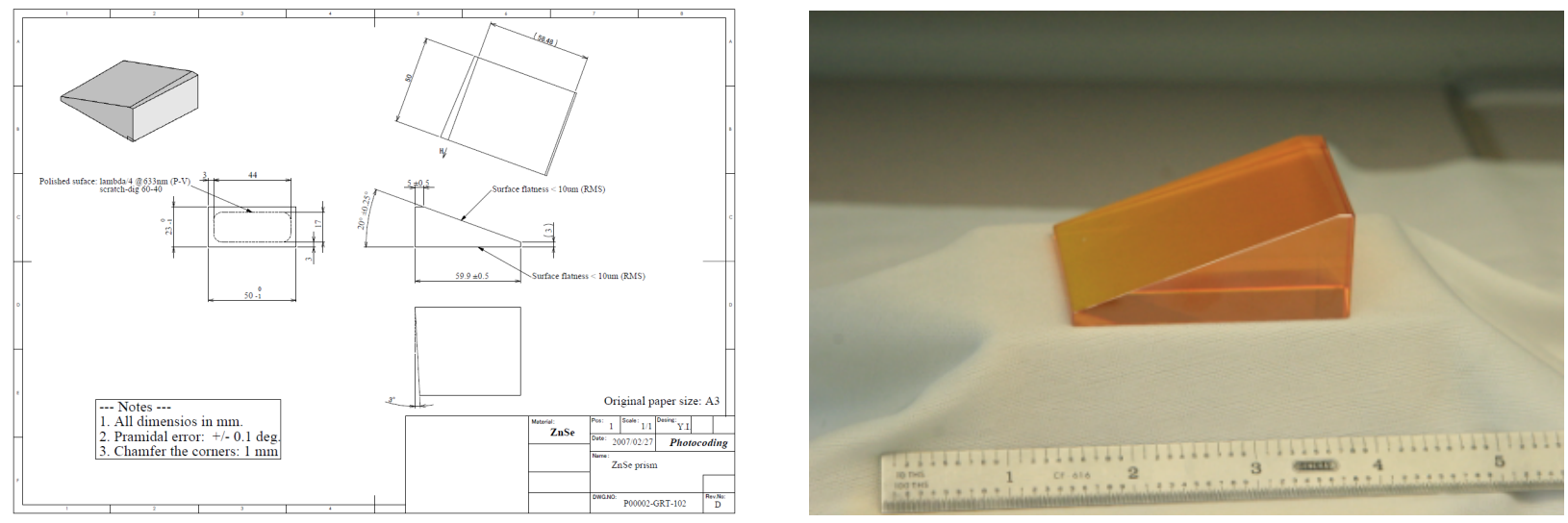

Figure 2. Drawing (left) and picture (right) of fabricated ZnSe immersion grating.

National Laboratory (LLNL). ${ }^{15-17}$ PERL II has three axes, and one of them rotates the spindle for cutting and the remaining two axes are for the linear stages in the orthogonal directions. The spindle speed and the feed rate were decided to $1000 \mathrm{rpm}$ and 0.4 or 0.35 inch per minute from the past experiments.

Before now, we fabricated three prism gratings during two years. The first sample showed the large wavefront aberration $(>5 \lambda$ in pv) in the diffracted light because of the degradation of the thermal control system. For the second one, this wavefront error problem was considerably improved but a lot of chippings were observed at the edge of the groove, which had not been seen in the previous experiences. As a result of some investigations, this might be thought to be caused by the subsurface damages produced by the first trial (We used the same prism to the first cutting after the groove surface was removed to be re-polished). For the third one, we can succeeded to reduce the chipping by completely removing and re-polishing the groove surface. However, unfortunately, owing to the trouble in the nano-positioner with the laser interferometer, the machine was halted and the cutting was not completed. Therefore, we investigated the optical performances of the second trial one. You can refer to Kuzmenko et al. (2010) ${ }^{18}$ for the more details about each trial and the results for the machining.

\section{OPTICAL TESTING AND PERFORMANCE OF THE PRISM GRATING}

We carried out optical testings to investigate four items, the wavefront error, the blaze angle, the random pitch error, and the grating ghost, for the second sample. We summarize the specifications and the results in Table.1. The details of the testings are described in the following subsections.

Table 1. Summary of optical specifications for the ZnSe immersion grating for WINERED and current performances of the second prism shaped grating.

\begin{tabular}{lcc}
\hline items & specifications & current resuts \\
\hline \hline groove pitch & $30 \mu \mathrm{m}$ & $30 \mu \mathrm{m}$ \\
blaze angle & $70 \pm 0.3 \mathrm{deg}$ & $66.91 \pm 0.15 \mathrm{deg}$ \\
surface irregularity (pv) & $\leq 0.63 \lambda$ at $0.633 \mu \mathrm{m}$ within $70 \mathrm{~mm}$ circle & $0.74 \lambda$ \\
surface irregularity (rms) & $\leq 0.045 \lambda$ at $0.633 \mu \mathrm{m}$ within $7.5 \mathrm{~mm}$ circle & $0.074 \lambda$ \\
surface roughness & $\leq 3.2 \mathrm{~nm}$ & $\leq 10.5 \mathrm{~nm}(\mathrm{rms})$ \\
random pitch error & $\leq 3.6 \mathrm{~nm}$ & $\leq 5.2 \mathrm{~nm}(\mathrm{rms})$ \\
ghost intensity & $\leq 0.1 \%$ & $20-40 \%$ \\
\hline
\end{tabular}

\subsection{Wavefront error}

The wavefront error in the diffracted beam is one of important specifications, because it determines the actual spectral resolution and spatial resolution of the spectrograph. The wavefront error of the WINERED immersion 
grating must be less than $0.63 \lambda(\mathrm{pv})$ for a $70 \mathrm{~mm}$ diameter and be less than $0.045 \lambda$ (rms) for a $7.5 \mathrm{~mm}$ diameter at $0.633 \mu \mathrm{m}$ (see Kuzmenko et al. $2010^{18}$ in more detail). Figure.3 shows the measured result of the wavefront error by Zygo interferometer. From the result of reanalysis, it is found that the final wavefront error is $0.74 \lambda$ (pv) with $70 \mathrm{~mm}$ diameter and $0.074 \lambda(\mathrm{rms})$ with $7.5 \mathrm{~mm}$ diameter, which closely satisfies the specification. As mentioned in the previous section, the wavefront error is considerablely sensitive to the temperature control in the enclosure of PERL II.

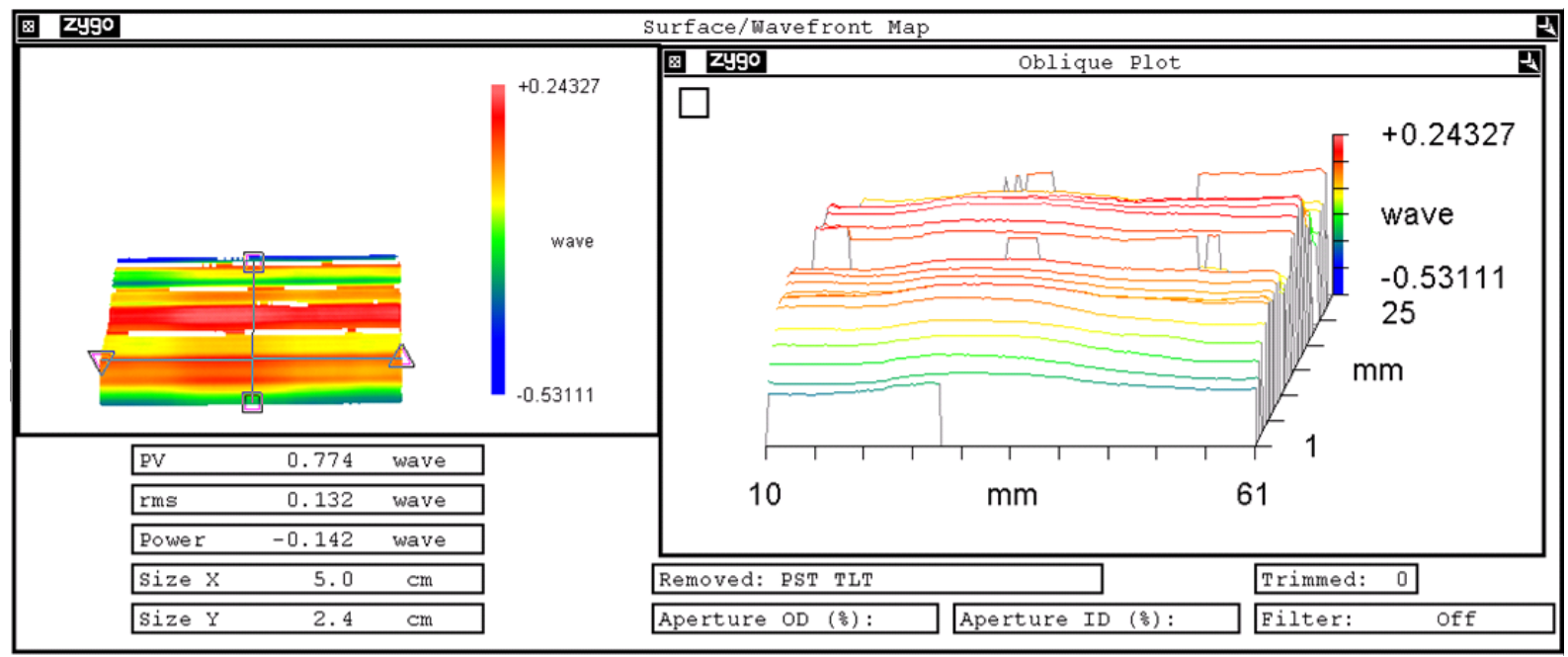

Figure 3. The wavefront error map of the cutting surface viewed from the Littrow position by Zygo interferometer. The unit wavelength is $0.633 \mu \mathrm{m}$.

\subsection{Blaze angle}

The blaze angle was measured with the similar method shown in Figure 6.8 of Loewen \& Popov (1991). ${ }^{19}$ At first, we prepared a $\mathrm{NeHe}$ laser and a rotational stage on which the grating is mounted using a specially designed jig. Second, the laser beam was turned normal to the base surface of the prism by rotating the stage $(\theta=0$ deg). Third, we searched two angles $\left(\theta_{1}\right.$ and $\left.\theta_{2}\right)$ at which the strongest and the second strongest orders go back to the exit hole of the laser, and measure their intensities $\left(I_{1}\right.$ and $\left.I_{2}\right)$ with a powermeter. Using these measured values, we can estimate the blaze angle with the following equation,

$$
\sin \theta_{\mathrm{B}}=\frac{I_{1} \sin \theta_{1}+I_{2} \sin \theta_{2}}{I_{1}+I_{2}} .
$$

We obtained both low and high blaze angles, which were $22.43 \pm 0.15 \mathrm{deg}$ and $66.91 \pm 0.15$ deg, respectively. From these angles, we can estimate the apex angle to be $90.66 \pm 0.21 \mathrm{deg}$ close to the right angle. The obtained high blaze angle differs from our requirement of $70 \pm 0.3 \mathrm{deg}$ by about $3 \mathrm{deg}$. These mean that the shape of one blaze is fabricated very well but the blaze unit could be slightly tilted from the base surface of the prism. Actually, it was found that there is a mis-alignment between the chip of tool and the base surface of PERL II. Before next fabrication, we have to carefully check and re-align the angle of tip of the tool, which is often different from that of the body of the tool.

\subsection{Random pitch error}

We can estimate the random pitch error from the integration of background scattered light among the diffraction orders (see Ikeda et al. 2008 ${ }^{14}$ ). To observe the faint scattered light sensitively, we built an optical train on the optical bench, with which the collimated laser beam was exposed to the grating and the diffracted spectra was taken by a cooled CCD camera (see the upper of Figure.4). The spectra obtained by this system is shown in Figure.5. The obtained scattered light relative to the diffracted main orders $\left(=I_{\mathrm{bg}} / \sum I\right)$, where $\sum I$ is the 
total intensity from main orders, was $3.0 \%$. In principle, the scattered light is mainly produced by the surface roughness and the random groove pitch error,

$$
\frac{I_{\mathrm{bg}}}{\sum I} \simeq \frac{I_{\mathrm{r}}}{\sum I}+\frac{I_{\mathrm{p}}}{\sum I},
$$

where $I_{\mathrm{r}} / \sum I$ and $I_{\mathrm{p}} / \sum I$ are scattered light generated by the surface roughness and the random pitch error. Then, each intensity for the grism configuration is given by

$$
\frac{I_{\mathrm{r}}}{\sum I}=\left(\frac{2 \pi(n-1) \sigma_{H}}{\lambda}\right)^{2},
$$

and

$$
\frac{I_{\mathrm{p}}}{\sum I}=\left(\frac{2 \pi(n-1) \sigma_{d} \sin \theta_{\mathrm{B}}}{\lambda}\right)^{2},
$$

when $\sigma_{H}$ and $\sigma_{d}$ are the surface roughness and the random pitch error in rms, respectively. We have obtained the surface roughness to be $10.5 \mathrm{~nm}$ (rms) by AFM profiler (see Table.1) which can produce the scattered light of $2.4 \%$ from Eq.(4). Therefore, the distribution of the random pitch error is at most $0.6 \%(=3.0 \%-2.4 \%) \dagger$. From Eq.(5), the equivalent random pitch errors is $\leq 5.2 \mathrm{~nm}(\mathrm{rms})$, which is agreement with the results for the past cutting using the ZnSe $\operatorname{disk}^{2}$ and closely satisfies the specification.

\subsection{Ghost}

There is no either Rowland ghost or Layman ghost, generated by periodic pitch errors, ${ }^{19}$ greater than $I_{\mathrm{gh}} / I_{0} \sim$ $10^{-3}$ in the spectrum of Figure.5. Instead, we can see prominent ghosts at the intermedium of orders, named as interorder ghost. The left of Figure.6 is a spectrum taken under the immersion configuration (see the lower of Figure.4). The intensities of interorder ghosts become much stronger than those for the grism configuration. These ghosts could be originated from a little bit something differences between odd and even grooves, attributed to the machining procedure. Because the grooves are cut by the tool while the x-stage on PERL II was moving in both-way (= bi-directional cutting in Figure.7), the groove pitch $(=d)$ and/or the shape of the blaze might differ between odd and even lines. These difference produce an alternative period with $2 d$, resulting in the interorder ghosts observed in the spectra. The right of Figure. 6 is a simulated spectrum if the centers of odd (or even) lines would be simultaneously shifted from their ideal positions by $0.4 \%$ of the groove pitch $(\Delta d / d=0.004)$ under the immersion configuration. This spectrum looks well reproducing the observed spectrum. For next cutting, we try the one-way cutting which could reduce these ghosts considerably (see Figure.7) because there is no difference among odd/even lines in principle.

\section{SELECTION OF REFLECTIVE COATING}

The normal incidence reflectivity of a ZnSe-air interface is about $18 \%$ at $\lambda=1 \mu \mathrm{m}$. The WINERED gratings require a groove reflectivity of at least $90 \%$. Hence, the grooves must receive a high reflectivity coating for the immersion grating to achieve a useful throughput. Previously, the issues of the reflective coating had been secondary to the more pressing problems of the cutting of the grating. But the coating is nontrivial and requires some attention.

A reflective coating for an immersion grating must accomplish several things. First it must be robust and adhere well to the substrate. Second it must have high reflectivity in immersion over the spectral range of interest. For a substrate of index $n_{I}$ and a coating of complex index $n_{\mathrm{II}}+i k_{\mathrm{II}}$, the immersed reflectivity $R$ is given by:

$$
R=\frac{\left(n_{\mathrm{I}}-n_{\mathrm{II}}\right)^{2}+k_{\mathrm{II}}^{2}}{\left(n_{\mathrm{I}}+n_{\mathrm{II}}\right)^{2}+k_{\mathrm{II}}^{2}} .
$$

\footnotetext{
${ }^{\dagger}$ Since the background scattered light could contain the other background source such as the bulk scattering suggested by Ikeda et al.(2009), ${ }^{20}$ the calculated value should be considered as the upper limit.
} 


\section{(a) Grism configuration}

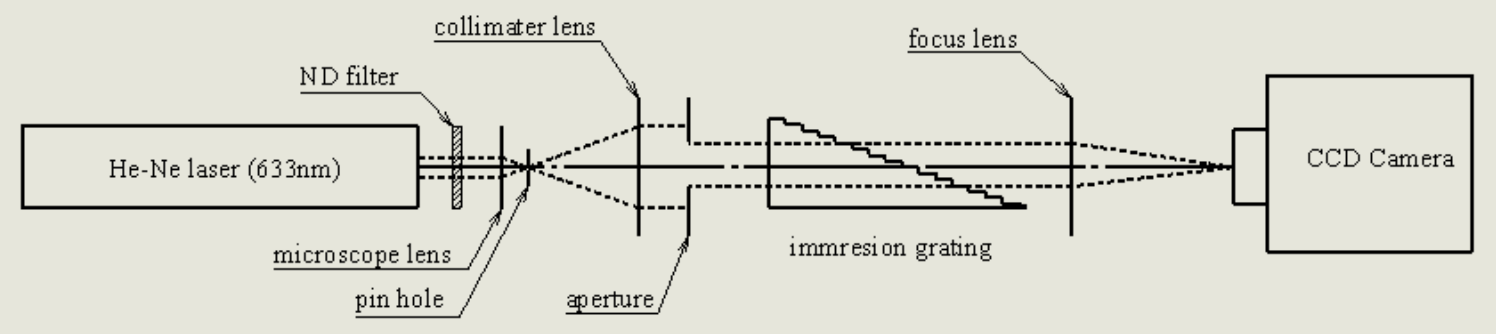

\section{(b) Immersion configuration}

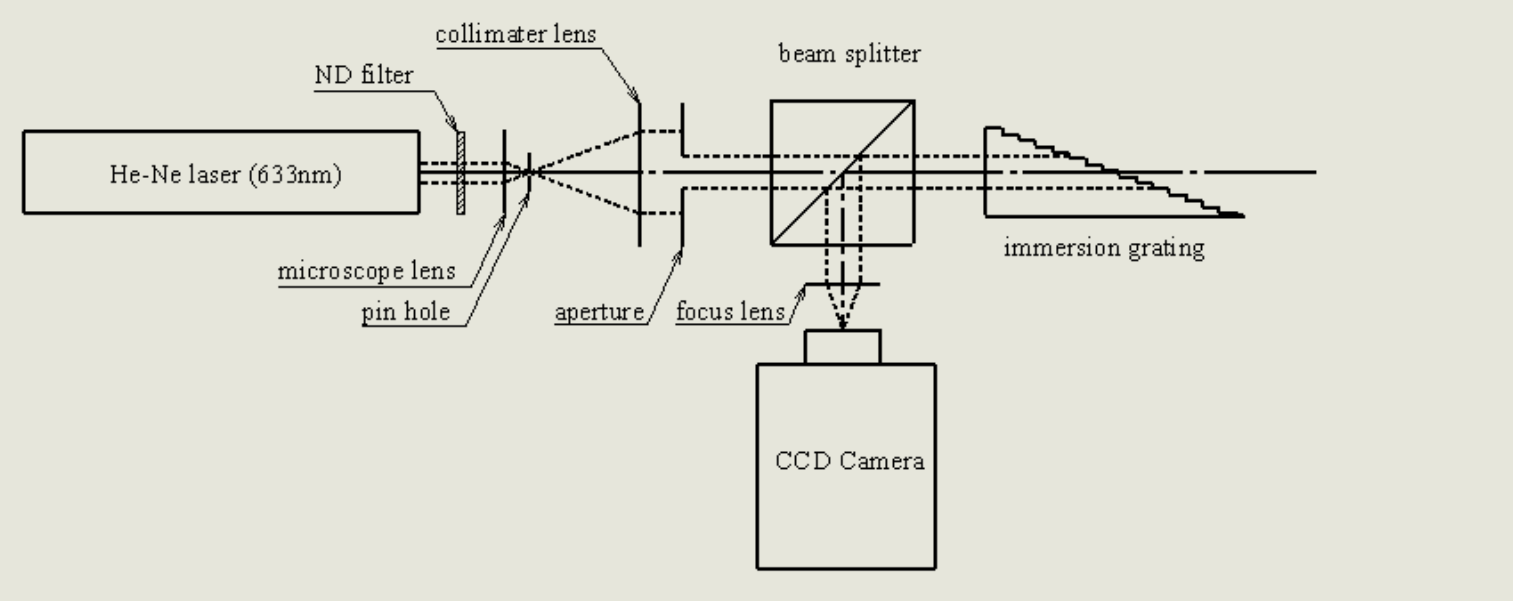

Figure 4. The optical configurations for the testing of the optical performances. (a) grism configuration: The HeNe laser beam is focused by the microscope lens, is cleaned by the spatial filter with the diameter of $15 \mu \mathrm{m}$, and is collimated again with the achromatic lens $(f=150 \mathrm{~mm})$. This beam is truncated by the $12 \mathrm{~mm}$ aperture and enters to the grating. The transmitting diffracted-light by the grating is focused by the camera lens $(f=80 \mathrm{~mm})$ to the cooled CCD camera. (b) immersion configuration: The collimation beam with the diameter $12 \mathrm{~mm}$, produced by the same optics to the grism configuration, enters the immersion grating. The reflective diffracted-light goes back, is bent to the right by the beam splitter, and is focused to the cooled CCD camera. 

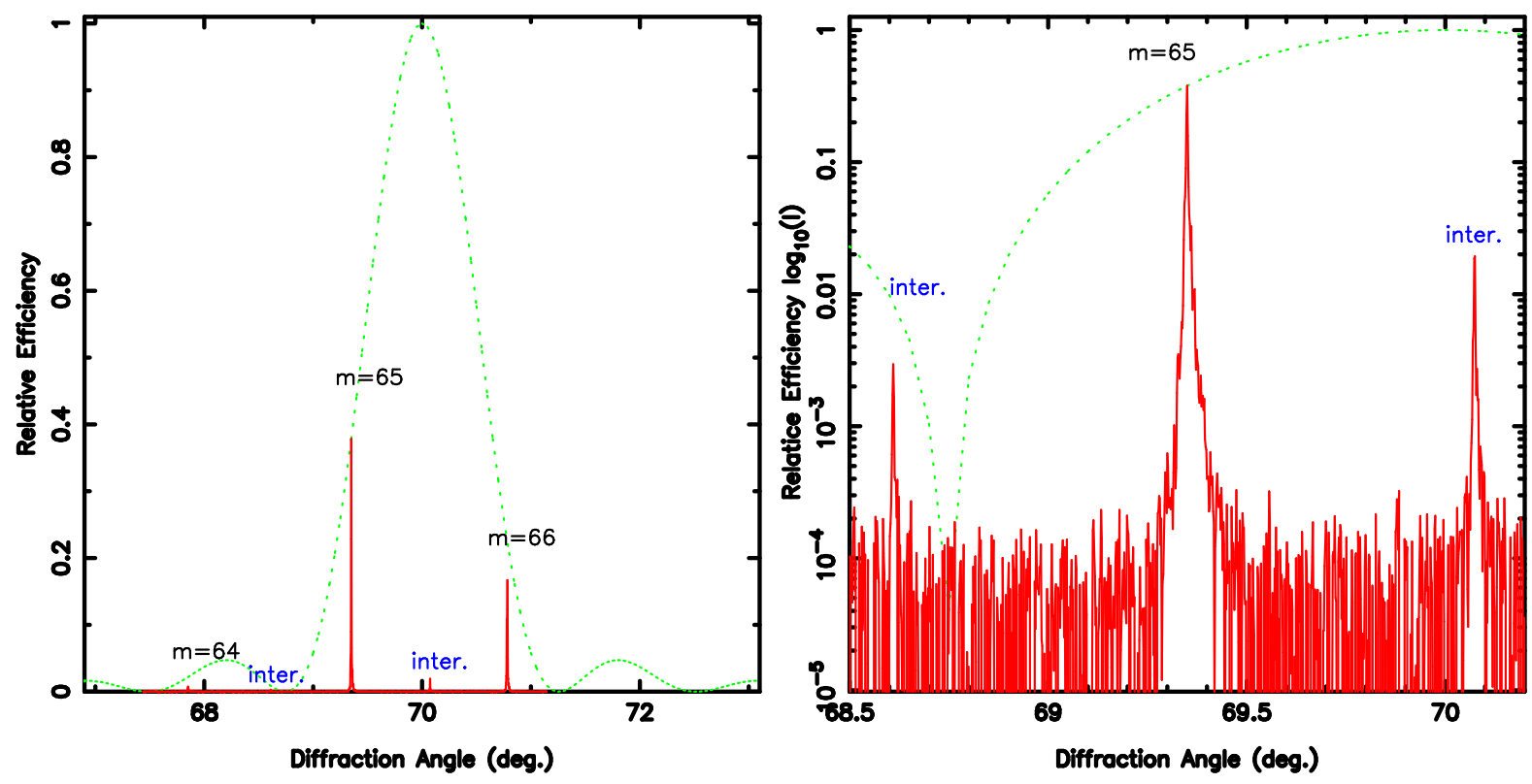

Figure 5. The HeNe laser spectrum in the grism configuration. Left is linear scale and Right is logarithm scale around the main order $(m=65)$. The dotted line show the blaze function by Fraunhofer diffraction theory.
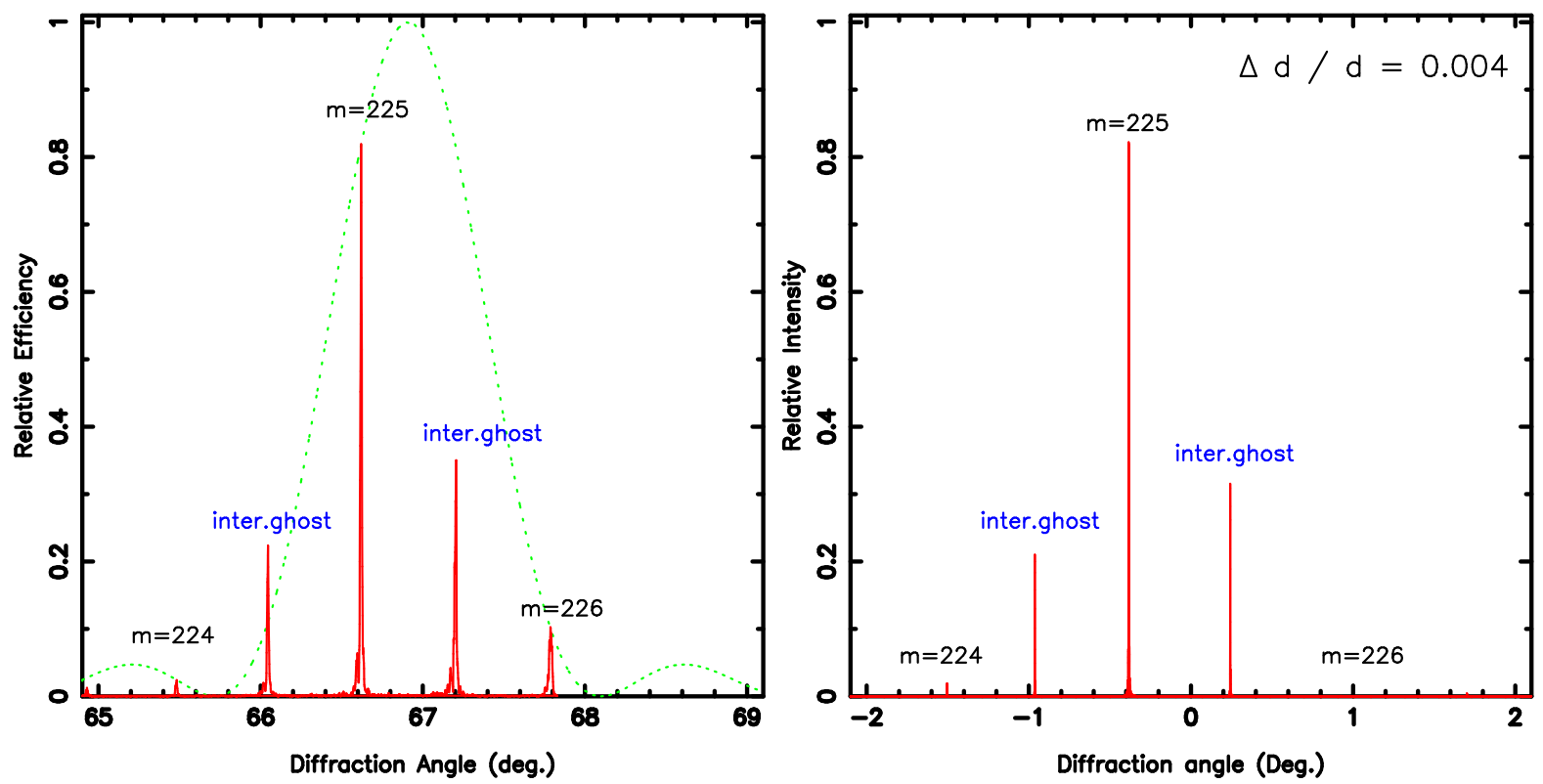

Figure 6. Left: The HeNe laser spectrum taken under the immersion configuration. Right: The simulated spectrum, assuming that the centers of the odd (or even) lines simultaneously shift from the the ideal position by $\Delta d=0.004$ (see text in more detail). We can see similar prominent inter-oder ghosts observed in the left spectrum.

Since $n_{\mathrm{I}}>1$, the immersed reflectivity will be lower than that of the reflectivity in air. Thirdly the coating should not increase the aberration in the diffracted wave. Finally the coating must deposit uniformly on the inclined and possibly shadowed surfaces of the grooves.

We limit our consideration to metal coatings for several reasons. The main one is broad spectral coverage. It is possible to achieve broadband reflectivity with dielectric coatings with many layers, however it often shows 


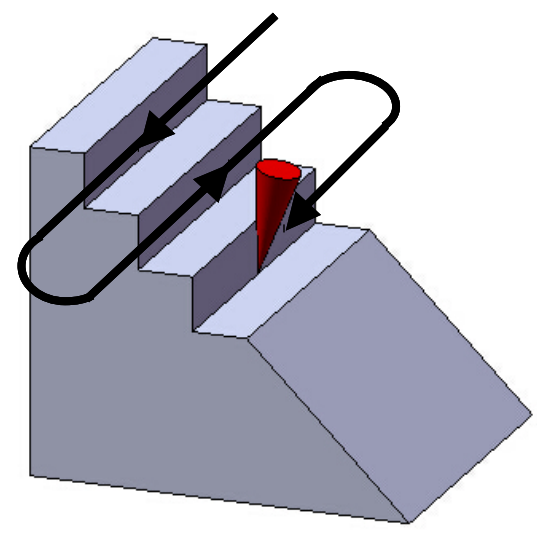

\section{bi-directional cutting}

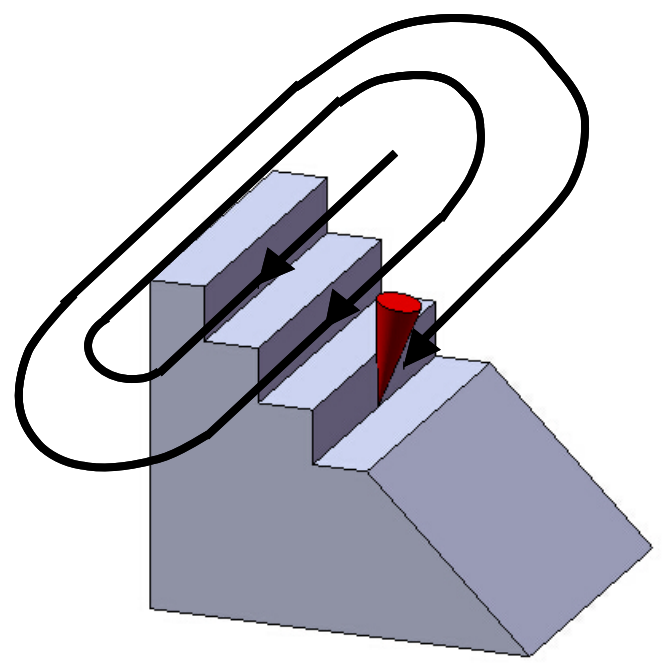

one-way cutting

Figure 7. The difference of cutting procedure. The left is bi-directional cutting. Because the tool moves in both ways, the difference of pitch or shape of the blazes could appear between odd and even lines. The right is one-way cutting. With this procedure, the ghost seen in the optical laser could be considerably reduced although it takes much time to machine the grating rather than the current one, resulting in necessity of more careful control of the temperature of PERL II.

the high frequent ripple in the spectra, which could degrade the photometric accuracy for high resolution spectroscopy. The second one is the thickness. While $100 \mathrm{~nm}$ is more than adequate for metals, several microns is needed for di-electronic coating. Thinner coatings result in lower coating stress and better adhesion. Furthermore the grating geometry can lead to shadowing effects if the coating material originates from a small area because of the thickness. The third one is the tolerance in thickness. Metal coatings have a high tolerance to variations in thickness. Once the thickness exceeds several optical depths the reflectivity remains constant. In the other hand, this is not the case for dielectric layers whose thickness must be tightly and controlled on the inclined surfaces and uniformity must be achieved for the wide field.

A search of the literature shows that no one has attempted to make an immersed mirror on ZnSe. However various metals have been deposited on ZnSe in attempts to make reliable electrical contacts. ${ }^{21,22}$ Useful information on adhesion can be gleaned from these works. Metals have also been used for electrical contacts of GaAs waveguide lasers, where the metal is part of the waveguide and affects the optical loss. ${ }^{23}$

Consider four common metals with known high reflectivity: $\mathrm{Al}, \mathrm{Ag}, \mathrm{Au}$, and $\mathrm{Cu}$. From their complex refractive indices ${ }^{24}$ one can calculate normal incidence reflectivity both in air and immersed in ZnSe (see Figure.8). Aluminium is the most common and therefore reliable material for the reflective coating. The University of Texas applies $2 \mu \mathrm{m}$ thick aluminum coatings on their silicon gratings and has been demonstrated to perform well from 1.1 to $5.5 \mu \mathrm{m} .{ }^{13} \mathrm{In}$ fact, we also made a sample (Al on a ZnSe disk with $25 \mathrm{~mm}$ diameter and $3 \mathrm{~mm}$ thickness) by suppering deposition method and confirmed that it closely shows the theoretical curve (Figure.8). Unfortunately, however, there is a problem with aluminum is that its reflectivity drops off below $1.1 \mu \mathrm{m}$. Thus, the aluminium is the baseline of use for ZnSe immersion grating of WINERED. Pure gold offers a high theoretical reflectivity but is known to have very poor adhesion to ZnSe. ${ }^{21}$ This is because gold is in phase equilibrium with ZnSe and there is no chemical reaction to enhance bonding. ${ }^{22}$ To make gold adhere one needs an intermediate thin-layer of a reactive metal like $\mathrm{Cr}$, as used for $\mathrm{Si}$ immersion grating $(3 \text { to } 5 \mu \mathrm{m})^{25}$ and Ge immersion grating $(8 \text { to } 13 \mu \mathrm{m})^{26}$ at LLNL. However, As can be seen in the right of Figure.8, $5 \mathrm{~nm}$ of Cr lowers the reflectivity to $<75 \%$. To achieve $90 \%$ reflectivity the thickness must be held to $<1 \mathrm{~nm}$, which is very difficult especially on steeply inclined surfaces. Turning to silver and copper, both are known to react at room temperature with the selenium ${ }^{27}$ in 

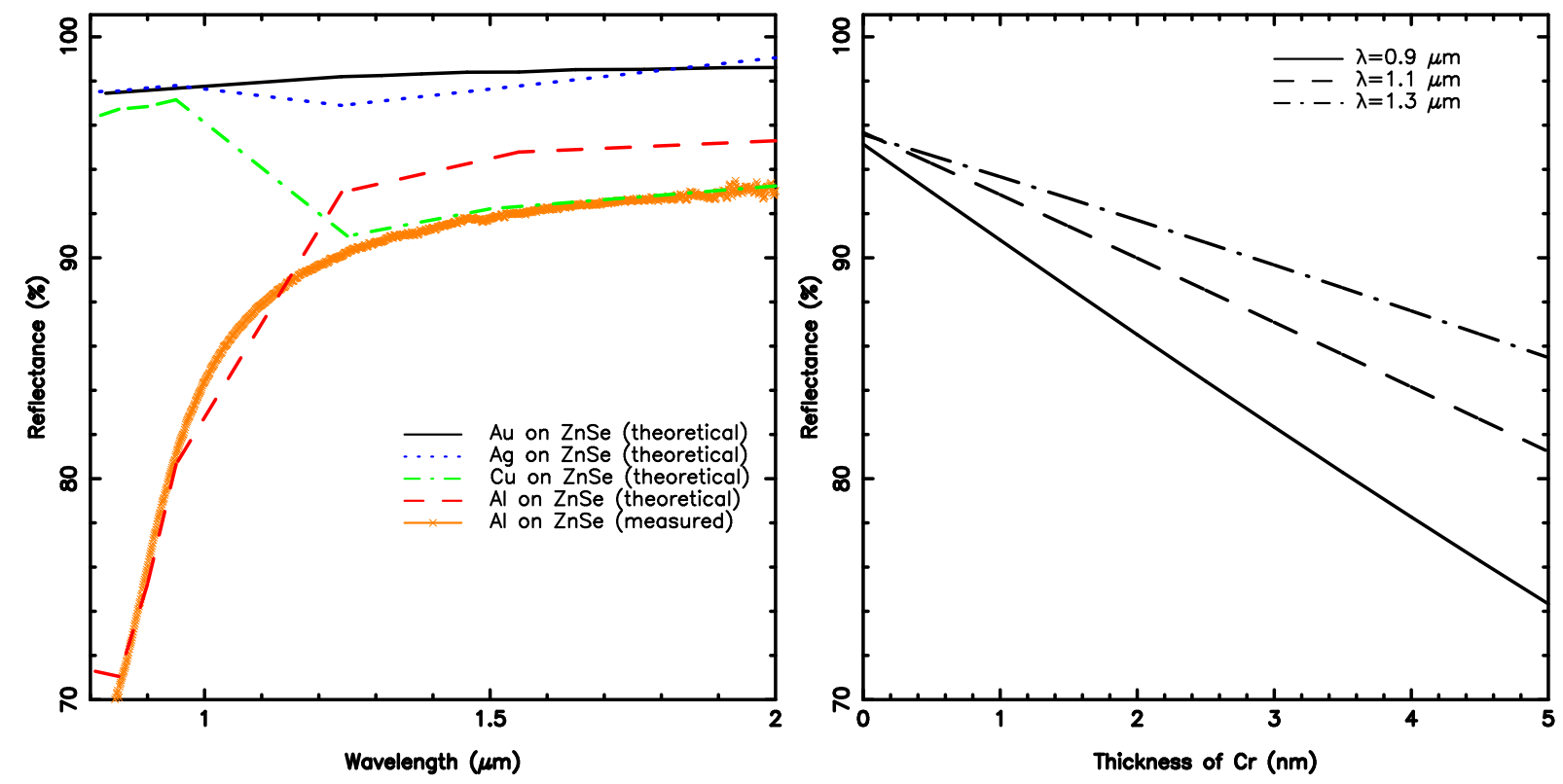

Figure 8. Internal reflectance of ZnSe substrate using various metals. Left: The calculated wavelength dependences for $\mathrm{Au}$ (solid curve), Ag (dotted curve), $\mathrm{Cu}$ (dot-dashed curve), and $\mathrm{Al}$ (Dashed curve), and a measured result of a sample $\mathrm{Al}$ coating by suppering deposition (cross marks: see the text in more detail). Right: The calculated thickness dependence of $\mathrm{Cr}$ immersed between Au layer and ZnSe substrate for three wavelengths $(0.9 \mu \mathrm{m}$ : solid curve, $1.1 \mu \mathrm{m}$ : dashed curve, and $1.3 \mu \mathrm{m}$ : dash-dotted curve).

ZnSe to form silver or copper selenide. This implies good adhesion and indeed this has been observed. The selenide should have little absorption in the few $\mathrm{nm}$ thick layers that may be formed. However, since the silver is easy to get oxidized in the air, the cupper is more practicable than the silver for WINERED. We plan to make the samples and measure the reflectivity of these combinations.

\section{SUMMARY AND FUTURE WORK}

We carried out first trials of fabrication of large prism shaped ZnSe immersion gratings using the nano precision fly cutting technique at Lawrence Livermore National Laboratory. We examined optical testings for the best one of those gratings, which closely satisfied the specifications for use of the near-IR high resolution spectrograph (=WINERED), except for the strong ghost see in the He-Ne laser spectrum under the immsersion configuration. This strong interorder ghost would be originated from the difference of pitch and/or shape of odd/even lines, which is attributed to the current cutting procedure "the bidirectional cutting". However, we could overcome this by "one-way cutting" procedure for next trial. In addition, we also investigated the suitable reflective coating on the groove surface of $\mathrm{ZnSe}$. As a result, we concluded that aluminum or cupper is the best material for WINERED wavelengths between 0.9 and $1.4 \mu \mathrm{m}$. The test coating of aluminum by suppering process shows the good reflectivity, which differs from the theoretical one by less than $2 \%$.

We plan the next cutting trail this summer. By improving the cutting process and additional R\&Ds about the coating, we could obtain a satisfied ZnSe prism grating by the beginning of 2011 .

\section{ACKNOWLEDGMENTS}

We would like to thank A. Tokunaga and J. Rayner for fruitful suggestions and comments through this project. The fabrication of the grating was performed under the auspices of the U. S. Department of Energy by Lawrence Livermore National Laboratory under Contract DE-AC52-07NA27344. This study was supported by KAKENHI(16684001) Grant-in-Aid for Young Scientists (A), KAKENHI(20340042) Grant-in-Aid for Scientific Research (B), and KAKENHI(21840052) Grant-in-Aid for Young Scientists (Start-up), by Japan Society for the 
Promotion of Science. It was also supported by the Grant for the Space Instrument Basic Development (FY2007 and FY2008) by ISAS. S.K. and C.Y. were financially supported by the Japan Society for the Promotion of Science (JSPS) during their graduate school days (-FY2009).

\section{REFERENCES}

[1] Kuzmenko, J. P., Little, S. L., Ikeda, Y., and Kobayashi, N., "Fabrication and testing of diamond-machined gratings in $\mathrm{ZnSe}, \mathrm{GaP}$, and bismuth germanate for the near infrared and visible," in [Advanced Optical and Mechanical Technologies in Telescopes and Instrumentation. Proceedings of the SPIE, 7018-181], Presented at the Society of Photo-Optical Instrumentation Engineers (SPIE) Conference 7018 (June 2008).

[2] Ikeda, Y., Kobayashi, N., Kuzmenko, P., Little, S., Yasui, C., Kondo, S., Minami, A., and Motohara, K., "Diamond-machined ZnSe immersion grating for NIR high-resolution spectroscopy," in [Advanced Optical and Mechanical Technologies in Telescopes and Instrumentation. Proceedings of the SPIE, 7018-183], Presented at the Society of Photo-Optical Instrumentation Engineers (SPIE) Conference $\mathbf{7 0 1 8}$ (June 2008).

[3] Yasui, C., Ikeda, Y., Kondo, S., Motohara, K., and Kobayashi, N., "WINERED: optical design of warm infrared echelle spectrograph," in [Ground-based and Airborne Instrumentation for Astronomy. Edited by McLean, Ian S.; Iye, Masanori. Proceedings of the SPIE, Volume 6269, pp. 62694P (2006).], Presented at the Society of Photo-Optical Instrumentation Engineers (SPIE) Conference 6269, 62694P (July 2006).

[4] Sebring, T. A., Moretto, G., Bash, F. N., Ray, F. B., and Ramsey, L. W., "The Extremely Large Telescope (ELT), a scientific opportunity; an engineering certainty," in [Proceedings of the Backaskog workshop on extremely large telescopes], Andersen, T., Ardeberg, A., and Gilmozzi, R., eds., 53 (2000).

[5] Monnet, G. and Gilmozzi, R., "Status of the ESO ELT," in [The Scientific Requirements for Extremely Large Telescopes], Whitelock, P., Dennefeld, M., and Leibundgut, B., eds., IAU Symposium 232, 429-431 (2006).

[6] Gilmozzi, R. and Spyromilio, J., "The European Extremely Large Telescope (E-ELT)," The Messenger 127, 11-+ (Mar. 2007).

[7] Nakagawa, T., "SPICA mission for mid- and far-infrared astronomy," in [Space Telescopes and Instrumentation III: Optical, Infrared, and Millimeter. Proceedings of the SPIE, 7010-16], Presented at the Society of Photo-Optical Instrumentation Engineers (SPIE) Conference 7010 (June 2008).

[8] Matsuhara, H. and Kataza, H., "Focal plane instruments onboard SPICA," in [Space Telescopes and Instrumentation III: Optical, Infrared, and Millimeter. Proceedings of the SPIE, 7010-18], Presented at the Society of Photo-Optical Instrumentation Engineers (SPIE) Conference 7010 (June 2008).

[9] Kobayashi, N., Ikeda, Y., Kawakita, H., Enya, K., Nakagawa, T., Kataza, H., Matsuhara, H., Hirahara, Y., and Tokoro, H., "Mid-infrared high-resolution spectrograph for SPICA," in [Society of Photo-Optical Instrumentation Engineers (SPIE) Conference Series], Presented at the Society of Photo-Optical Instrumentation Engineers (SPIE) Conference $\mathbf{7 0 1 0}$ (Aug. 2008).

[10] Sarugaku, Y., Kobayashi, N., Ikeda, Y., Enya, K., Kataza, H., Nakagawa, T., Matsuhara, H., Kawakita, H., Hirahara, Y., and Tokoro, H., "Mid-IR high-resolution Echelle spectrometer (MIRHES) for SPICA," SPIE Proc. 7731 (2010).

[11] Ikeda, Y., Kobayashi, N., Kondo, S., Yasui, C., Motohara, K., and Minami, A., "WINERED: a warm near-infrared high-resolution spectrograph," in [Ground-based and Airborne Instrumentation for Astronomy. Edited by McLean, Ian S.; Iye, Masanori. Proceedings of the SPIE, Volume 6269, pp. 62693T (2006).], Presented at the Society of Photo-Optical Instrumentation Engineers (SPIE) Conference 6269, 62693T (July 2006).

[12] Yasui, C., Ikeda, Y., Kondo, S., Motohara, K., Minami, A., and Kobayashi, N., "Warm infrared Echelle spectrograph (WINERED): testing of optical components and performance evaluation of the optical system," in [Ground-based and Airborne Instrumentation for Astronomy II. Proceedings of the SPIE, 7014-108], Presented at the Society of Photo-Optical Instrumentation Engineers (SPIE) Conference 7014 (June 2008).

[13] Marsh, J. P., Mar, D. J., and Jaffe, D. T., "Production and evaluation of silicon immersion gratings for infrared astronomy," Applied Optics 46, 3400-3416 (June 2007). 
[14] Ikeda, Y., Kobayashi, N., Terada, H., Shibayama, A., Ozawa, A., Yasui, C., Kondo, S., Pyo, T., and Kawakita, H., "High-efficiency silicon immersion grating by electron-beam lithography for high-resolution NIR spectroscopy," in [Ground-based and Airborne Instrumentation for Astronomy II. Proceedings of the SPIE, 7014-226], Presented at the Society of Photo-Optical Instrumentation Engineers (SPIE) Conference 7014 (June 2008).

[15] Kuzmenko, P. J., Davis, P. J., Little, S. L., and Hale, L. C., "Materials and fabrication issues for large machined germanium immersion gratings," in [Optomechanical Technologies for Astronomy. Edited by AtadEttedgui, Eli; Antebi, Joseph; Lemke, Dietrich. Proceedings of the SPIE, Volume 6273, pp. 62732W (2006).], Presented at the Society of Photo-Optical Instrumentation Engineers (SPIE) Conference 6273, 62732W (July 2006).

[16] Kuzmenko, P. J., Davis, P. J., Little, S. L., Little, L. M., and Bixler, J. V., "High efficiency germanium immersion gratings," in [Optomechanical Technologies for Astronomy. Edited by Atad-Ettedgui, Eli; Antebi, Joseph; Lemke, Dietrich. Proceedings of the SPIE, Volume 6273, pp. 62733T (2006).], Presented at the Society of Photo-Optical Instrumentation Engineers (SPIE) Conference 6273, 62733T (July 2006).

[17] Kuzmenko, P. J., "Prospects for machined immersion gratings in the near infrared and visible," in [Optomechanical Technologies for Astronomy. Edited by Atad-Ettedgui, Eli; Antebi, Joseph; Lemke, Dietrich. Proceedings of the SPIE, Volume 6273, pp. 62733S (2006).], Presented at the Society of Photo-Optical Instrumentation Engineers (SPIE) Conference 6273, 62733S (July 2006).

[18] Kuzmenko, P. J., Little, L. M., Ikeda, Y., and Kobayashi, N., "Progress in the fabrication of a prototype ZnSe immersion grating for the WINERED spectrograph," SPIE Proc. this volume (2010).

[19] Loewen, G. E. and Popov, E., eds., [Difftraction gratings and applications ], Marcel Dekker Inc., New York (1991).

[20] Ikeda, Y., Kobayashi, N., Kondo, S., Yasui, C., Kuzmenko, P. J., Tokoro, H., and Terada, H., "Zinc sulfide and zinc selenide immersion gratings for astronomical high-resolution spectroscopy: evaluation of internal attenuation of bulk materials in the short near-infrared region," Optical Engineering 48, 084001-+ (Aug. 2009).

[21] Tadanaga, O., Koide, Y., Hashimoto, K., Oku, T., Teraguchi, N., Tomomura, Y., Suzuki, A., and Murakami, M., "Dependence of electrical properties on work functions of metals contacting to p-type ZnSe," Japanese Journal of Applied Physics 35, 1657-1663 (1996).

[22] Goesmann, F., Mölle, M., Studnitzky, T., and Schmid-Fetzer, R., "Interface reactions and electrical properties of metal contacts (Ti, In, Au, W) on p-ZnSe," Semiconductor Science Technology 13, $236-240$ (Feb. 1998).

[23] Smith, G. M., Forbes, D. V., Lammert, R. M., and Coleman, J. J., "Metallization to asymmetric cladding separate confinement heterostructure lasers," Applied Physics Letters 67, 3847-3849 (Dec. 1995).

[24] Ordal, M. A., Long, L. L., Bell, R. J., Bell, S. E., Bell, R. R., Alexander, Jr., R. W., and Ward, C. A., "Optical properties of the metals $\mathrm{Al}, \mathrm{Co}, \mathrm{Cu}, \mathrm{Au}, \mathrm{Fe}, \mathrm{Pb}, \mathrm{Ni}, \mathrm{Pd}, \mathrm{Pt}, \mathrm{Ag}, \mathrm{Ti}$, and $\mathrm{W}$ in the infrared and far infrared," Applied Optics 22, 1099-1119 (Apr. 1983).

[25] Kuzmenko, P. J., Ciarlo, D. R., and Stevens, C. G., "Fabrication and testing of a silicon immersion grating for infrared spectroscopy," in [Society of Photo-Optical Instrumentation Engineers (SPIE) Conference Series], J. Wang \& P. B. Hays, ed., Society of Photo-Optical Instrumentation Engineers (SPIE) Conference Series 2266, 566-577 (Sept. 1994).

[26] Kuzmenko, P. J., Little, L. M., Davis, P. J., and Little, S. L., "Modeling, fabrication, and testing of a diamond-machined germanium immersion grating," SPIE Proc. 4850, 1179-1190 (2003).

[27] Ouchi, A., Bastl, Z., Bohacek, J., Orita, H., Miyazaki, K., Miyashita, S., Bezdicka, P., and Pola, J., "Roomtemperature reaction between laser chemical vapor deposited selenium and some metals," Chemistry of Materials 16, 3439-3445 (2004). 